\section{(A) Check for updates}

Cite this: Dalton Trans., 2018, 47 8605

Received 2nd October 2017 Accepted 17th January 2018 DOI: $10.1039 / c 7 d t 03698 a$ rsc.li/dalton

\title{
Stimuli-responsive protection of optically excited triplet ensembles against deactivation by molecular oxygen $\uparrow$
}

\author{
N. V. Nazarova, (D) ${ }^{a}$ Yu. S. Avlasevich, (D) ${ }^{a}$ K. Landfester (D) a and S. Baluschev (D) ${ }^{a}$, b \\ Herein we demonstrate temperature-dependent sacrificial singlet oxygen scavenging properties of \\ $\mathrm{N}$-butyl-2-pyridone, ensuring efficient stimuli-responsive protection of densely populated excited triplet \\ state ensembles against deactivation by molecular oxygen. As an acting external stimulus the temperature \\ was chosen: it will be shown that at low temperature the concentration of singlet oxygen will be substan- \\ tially lowered; in contrast, at elevated temperatures singlet oxygen will not be captured, and thus the opti- \\ cally excited densely populated triplet ensembles will be effectively depopulated. The singlet oxygen \\ scavenging ability of $\mathrm{N}$-butyl-2-pyridone demonstrates long-term protection of a triplet-triplet annihil- \\ ation upconversion process against photooxidation.
}

\section{Introduction}

The triplet-triplet annihilation photon energy upconversion process (TTA-UC) relies on optically created densely populated organic triplet ensembles, in which the inter-molecular energy transfer depends strongly on a variety of environmental parameters, such as temperature or contamination with molecular oxygen. The TTA-UC takes place in multi-chromophore systems consisting of energetically optimized pairs of emitter molecules (typically aromatic hydrocarbons) and sensitizer molecules (metallated macrocycles, ${ }^{1}$ such as porphyrins and phthalocyanines).

Metallated porphyrins are widely used for thermal- or oxygen sensing, ${ }^{2}$ where the dependence of integral phosphorescence emission or the decay time of the phosphorescence is registered. These sensing processes involve optical excitation of the metallated macrocycle molecule and registration of the decreased phosphorescence or phosphorescence decay time as a function of the increased sample temperature or oxygen content. $^{3}$

Oxygen sensors for detecting extremely low levels of oxygen i.e. physiologically relevant $\mathrm{O}_{2}$ concentrations (ranging from nM-200 $\mu \mathrm{M}$ ) deserve special attention. ${ }^{4}$ For example, one can mention the transient state imaging technique, ${ }^{5}$ single-nano-

\footnotetext{
${ }^{a}$ Max Planck Institute for Polymer Research, Ackermannweg 10, D-55128 Mainz, Germany.E-mail: balouche@mpip-mainz.mpg.de

${ }^{b}$ Optics and Spectroscopy Department, Faculty of Physics, Sofia University,

"St. Kliment Ochridski", 5 James Bourchier, 1164 Sofia, Bulgaria

$\dagger$ Electronic supplementary information (ESI) available. See DOI: 10.1039/ c7dt03698a
}

particles with a ratiometric $\mathrm{O}_{2}$-response ${ }^{6}$ or phosphorescent metalloporphyrins encapsulated in hydrophobic dendrimers. ${ }^{7,8}$ The main experimental drawback of these sensing techniques is that phosphorescence emission is an integral parameter, which depends simultaneously on the local temperature and local oxygen contamination.

The triplet-triplet annihilation photon energy upconversion process represents a thorough solution for the problem of oxygen sensing: instead of a single material response on the acting parameter (variation of the oxygen concentration), the TTA-UC provides a ratiometric material response. The use of the mutual dependence of the residual sensitizer phosphorescence and emitter delayed fluorescence on the acting parameter ensures inherent/instantaneous compensation regarding other unwanted local changes in the sample parameters. ${ }^{9}$ Another advantage of the TTA-UC as a sensing technique is its extremely low excitation intensity (less than $1-10 \mathrm{~mW} \mathrm{~cm}^{-2}$ ): efficient TTA-UC was observed even with non-coherent light $\left(\right.$ sunlight $\left.t^{10}\right)$. Application potential of the TTA-UC process ranges from the fields of molecular sensing ${ }^{11}$ and bioimaging, ${ }^{12}$ solid state optical technologies ${ }^{13}$ up to renewable energy sources. ${ }^{14}$

A simplified representation of the TTA-UC process is shown in Fig. 1. The chemical structures of the used sensitizer meso-tetraphenyl-tetrabenzo[2,3]porphyrin palladium(II) (PdTBP) and the emitter - 3,9(10)-bis(3,3-dimethylbutyn-1-yl) perylene (BDP) are shown as insets in Fig. 1. The photon energy is absorbed by the sensitizer (the green arrow, Fig. 1). The long-lived triplet state of the sensitizer is formed due to the efficient inter-system crossing (ISC) enhanced by the spinorbital coupling of the heavy metal centre. Furthermore, this 


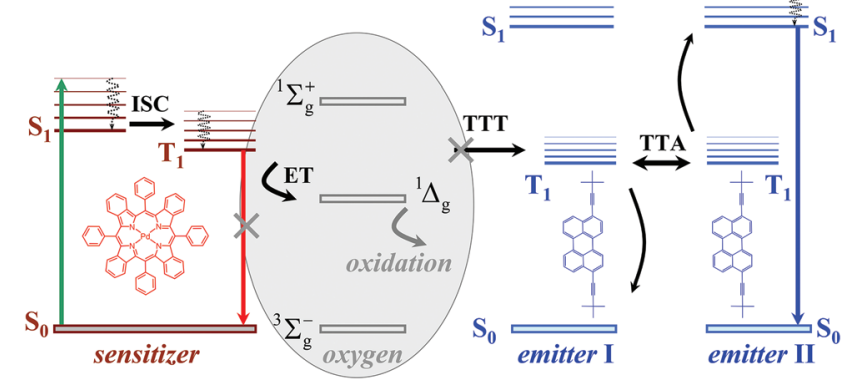

Fig. 1 Simplified energetic scheme of the triplet-triplet annihilation upconversion process in an oxygen contaminated environment, containing singlet oxygen scavenging moieties. Insets: Structure of the sensitizer (PdTBP) and emitter (BDP); SOS - singlet oxygen scavenger.

energy is transferred to an emitter triplet state via the triplettriplet transfer (TTT) process. Then two excited emitter molecules undergo triplet-triplet annihilation (TTA), in which one emitter molecule returns to its singlet ground state and the other molecule gains the energy of both triplet states and is excited to the higher singlet state. As the emitter singlet state decays radiatively back to the ground state, a delayed fluorescence photon (dark blue line, Fig. 1) bearing higher energy than that of the excitation photons is emitted. It is important to note that the sensitizer excited triplet state is not completely depopulated via the TTT process; therefore residual sensitizer phosphorescence is observed too (Fig. 1, red arrow).

The above described excitation energy transfers occur in an oxygen-free environment. In an environment contaminated with molecular oxygen ${ }^{15}$ even at the ppm-level, the TTA-UC process becomes more complicated. In the presence of molecular oxygen, the energy stored in the excited states of the triplet ensembles is actively dissipated, competing with emissive (phosphorescence) or non-emissive (triplet to triplet) energy transfer processes. The reason for this is the process of energy transfer between the excited organic triplet state and the ground state of molecular oxygen, followed by the generation of singlet oxygen $\left({ }^{1} \Delta_{\mathrm{g}}\right.$, Fig. 1$)$. Singlet oxygen is a highly reactive species, leading to the oxidation of the photoactive molecules, followed by further loss of efficiency. An extensive review ${ }^{16}$ describing the oxygen protection strategies of organic ensembles, undergoing triplet-triplet annihilation, has been recently published. Earlier reversible photoswitching and chemical-responsive TTA-UC were demonstrated. ${ }^{17}$

Herein the sensing portfolio of the TTA-UC process will be enriched - we demonstrate for the first time stimuli responsive protection of the upconverting organic ensemble. As an acting external stimulus we chose temperature: it will be shown that at low temperature the concentration of singlet oxygen will be substantially lowered; in contrast, at elevated temperatures the singlet oxygen will be not captured; thus the optically excited densely populated triplet ensembles will be effectively depopulated.

Polycyclic aromatic hydrocarbons are able to bind singlet oxygen forming the corresponding endoperoxides (EPOs). ${ }^{18}$
Recently, reversible singlet oxygen binding for optical and photo-medical applications was reviewed. ${ }^{19}$ The endoperoxide formation is strongly temperature dependent. Furthermore, some EPOs exhibit the exceptional feature of releasing oxygen, frequently in the excited singlet state, ${ }^{20}$ under heating ${ }^{21}$ or UV irradiation. ${ }^{18}$ Thus the endoperoxide formation allows for keeping the singlet $\mathrm{O}_{2}$ concentration in the UC-ensemble at a certain level, co-regulated by the sample temperature. The fractional photodynamic therapy ${ }^{22}$ needs a sensitive technology, measuring the concentration of the singlet oxygen released during the "dark"-periods in an all-optical manner and under a change in the sample temperature: since the TTA-UC process demonstrates the necessary sensitivity and after the corresponding calibration, the TTA-UC can be used as such a sensing tool. The reported stimuli-responsive UC-process could be used as real-time control of the concentration of singlet oxygen, generated during the post-illumination period for the purpose of fractional photodynamic therapy. ${ }^{22}$ Even more, the proposed TTA-UC process is much more sensitive to the presence of singlet oxygen than the commonly used sensing process - phosphorescence.

The mechanism of the cycloaddition of ${ }^{1} \mathrm{O}_{2}$ to the aromatic compounds is very similar to the classical Diels Alder type reactions. The reactivity of aromatic hydrocarbons toward ${ }^{1} \mathrm{O}_{2}$ increases with the electron density of the aromatic substrate, reflecting the electrophilic nature of ${ }^{1} \mathrm{O}_{2} \cdot{ }^{18}$ The easiness of oxygen release from these systems depends on the polycyclic aromatic system and the nature of the substituents at the meso-positions. ${ }^{20}$

As the next step, it will be shown that $N$-butyl-2-pyridone (NBP) is capable of binding ${ }^{1} \mathrm{O}_{2}$ dissolved in an organic solvent in a temperature dependent manner. 2-Pyridone itself exists in two forms; the proton bound to nitrogen can migrate to oxygen, giving the second tautomer, 2-hydroxypyridine. ${ }^{23}$ The first form can be stabilized by $N$-alkylation; a long alkyl chain in such compounds facilitates their miscibility with nonpolar organic solvents. $N$-Alkyl-2-pyridones represent a stable diene structure, which can undergo [4 +2]-cycloaddition (Diels-Alder reaction) with different dienophiles, like alkenes ${ }^{24}$ or molecular oxygen in the singlet excited state. ${ }^{25,26}$ It is known that 2-pyridones bind singlet oxygen efficiently at lower temperatures and release it upon heating. ${ }^{25}$ Therefore, 2-pyridones were used for catalytic purposes, ${ }^{25,26}$ as well as prominent biological antifungal and antiviral agents. ${ }^{27}$

Irradiation of palladium benzoporphyrin (PdTBP) dissolved in a mixture of toluene $(80 \% \mathrm{vol})$ and NBP solution $(20 \% \mathrm{vol})$ leads to the formation of $N$-butyl-2-pyridone EPO. The reaction scheme is shown in Fig. 2. This reaction is strongly temperature dependent; therefore the endoperoxides decompose thermally and regenerate oxygen (probably in a singlet state, we did not study this process explicitly) and the parent hydrocarbon. The lifetime of the EPO depends strongly on the temperature. ${ }^{19}$ At low temperature singlet oxygen can be stored for prolonged periods of time and then released by decomposition upon heating; thus the bindingrelease cycle can be repeated. 


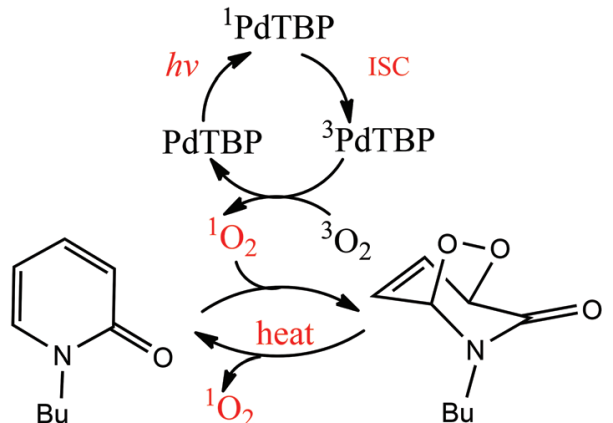

Fig. 2 Reversible addition of singlet oxygen to $\mathrm{N}$-butyl-2-pyridone. ${ }^{1} \mathrm{PdTBP}$ represents the first excited singlet state of the sensitizer (corresponds to excitation at the Q-band); ${ }^{3} \mathrm{PdTBP}$ represents the first excited triplet state of the sensitizer.

\section{Experimental}

\section{Materials}

The sensitizer - meso-tetraphenyl-tetrabenzo[2,3]porphyrin palladium(II) (PdTBP) ${ }^{12}$ and the emitter - 3,10-bis(3,3-dimethylbutyn-1-yl)perylene (BDP) ${ }^{28}$ were synthesized as described elsewhere. $N$-Butyl-2-pyridone was synthesized by alkylation of 2-hydroxypyridine with 1-bromobutane ${ }^{29}$ and purified via vacuum distillation.

For the samples prepared in a glove-box commercial solvents (ultra-dry, packed under argon, septum sealed) were used as received. NBP solutions were placed into a glove-box ( $4 \mathrm{ppm}$ of $\mathrm{O}_{2}$ ) and stirred in an open bottle for $2 \mathrm{~h}$; thus the residual oxygen contamination at the NBP corresponds to the glove-box oxygen content. Sample solutions were prepared and sealed in the glove-box. For all optical measurements, the molar concentrations of the sensitizer (PdTBP) and emitter (BDP) were the same, $1 \times 10^{-5} \mathrm{M} / 2 \times 10^{-4} \mathrm{M}$, respectively.

\section{Method}

Luminescence spectra were recorded by a home-made setup. Typically, sample was located in a temperature-controlled cuvette holder. The cuvette holder temperature was controlled with a Peltier element by means of the T-app computer program from Electron Dynamics Ltd (Southampton, UK). The sample temperature additionally was measured through a thermistor (PT100) attached on the top of the cuvette. The sample was excited with wavelength $\lambda_{\text {exc }}=633 \mathrm{~nm}$ light beam from a HeNe laser. The excitation power was controlled using a power meter PM 100D (Thorlabs, USA). For regular measurements, the excitation intensity was set to $12 \mathrm{~mW} \mathrm{~cm}^{-2}$ and the laser spot diameter was $d=2 \times 10^{-3} \mathrm{~m}$.

\section{Results}

Active protection of the densely populated triplet ensembles from deactivation by molecular oxygen is based on the application of oxygen scavenging species which react with singlet oxygen to minimize the amount of oxygen available for deteriorative reactions, leading to the degradation of the photoactive molecules. ${ }^{16}$ An evident drawback of this method is the possible unwanted interaction between the scavenger and the UC-active moieties. Therefore, to start with the influence of the NBP on the efficiency of the TTA-UC process was studied. The luminescence spectra of the studied UC-couple under glovebox conditions were compared (Fig. 3). Experimental conditions: samples prepared and sealed in a nitrogen filled glovebox (4 ppm $\mathrm{O}_{2}$ ); temperature $-25{ }^{\circ} \mathrm{C}$; excitation intensity $12 \mathrm{~mW} \mathrm{~cm}^{-2}$; HeNe laser; concentrations of the sensitizer (PdTBP) and emitter (BDP), $1 \times 10^{-5} \mathrm{M} / 2 \times 10^{-4} \mathrm{M}$, respectively.

As shown in Fig. 3a, the steady-state UC-signal in a solvent mixture containing $20 \%$ vol NBP is slightly lower (about 15\% less efficiency) than the UC-signal in toluene. The temporal dependence of the UC-signal for the pure toluene sample shows typical behaviour (Fig. 3b, black line) for the UCsamples in an environment with residual oxygen contamination (4 ppm $\mathrm{O}_{2}$ ): the UC-fluorescence rises during the first 10 seconds of optical excitation, until the complete oxygen content is consumed (for instance, by oxidation of the BDP emitter molecule ${ }^{30}$ ). In contrast, the UC-sample containing $20 \%$ vol NBP demonstrates fast growth (the integration time of the registration device is set to $100 \mathrm{~ms}$ ) because the concen-
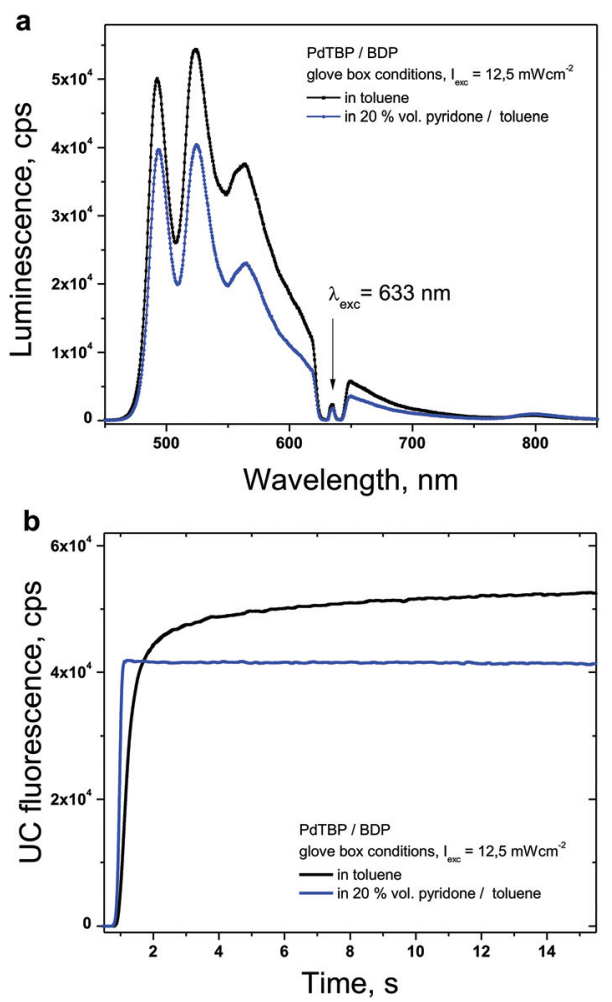

Fig. 3 (a) Luminescence spectra for the samples containing the UCcouple PdTBP/BDP, dissolved in toluene (black line); in a mixture of $\mathrm{N}$-butyl-2-pyridone/toluene (blue line). (b) Temporal dependences of the UC-signal at the fluorescence maximum $(\lambda=526 \mathrm{~nm})$. 
tration of the oxygen dissolved in the toluene/NBP solution is very low.

\section{Temperature effect}

The densely populated organic triplet ensemble participating in the TTA-UC process is created by optical excitation performed in the continuous wave (cw) regime. Therefore the delayed fluorescence of the emitter molecule (the UC-signal) the emission with a central wavelength around $526 \mathrm{~nm}$ (Fig. 4) and the residual phosphorescence of the sensitizer - the emission with a central wavelength around $800 \mathrm{~nm}$ (Fig. 4) can be observed simultaneously. In the environment contaminated with molecular oxygen an additional lost channel is present it is the generation of singlet oxygen (Fig. 2) leading to the modulation and loss of efficiency of both optical signals delayed fluorescence as well the residual phosphorescence. If a sacrificial singlet oxygen scavenger such as NBP is present, it is possible to bind a large amount of the present molecular oxygen, thus excluding it as a loss mechanism for the TTA-UC and phosphorescence processes. The usage of $N$-butyl-2-pyridone allows implementation of an additional acting parameter - since the probability of the creation of EPOs is temperature dependent, the rate in which the local amount of molecular oxygen will be bound will also be temperature dependent. In Fig. 4 the temperature dependence of the UC-fluorescence and the residual phosphorescence for samples protected by NBP is demonstrated. Experimental conditions: UC-couple PdTBP/ BDP, dissolved in a mixture of $20 \%$ vol $N$-butyl-2-pyridone/ toluene; excitation intensity $-12 \mathrm{~mW} \mathrm{~cm}^{-2}$; HeNe laser; concentration of the sensitizer (PdTBP) and emitter (BDP), $1 \times$ $10^{-5} \mathrm{M} / 2 \times 10^{-4} \mathrm{M}$, respectively; ambient environment (oxygen saturated solvents). The sample is continuously illuminated; the luminescence spectra were obtained at the 100th second after the start. For each temperature measurement a new sample point, with a large lateral displacement, was chosen.

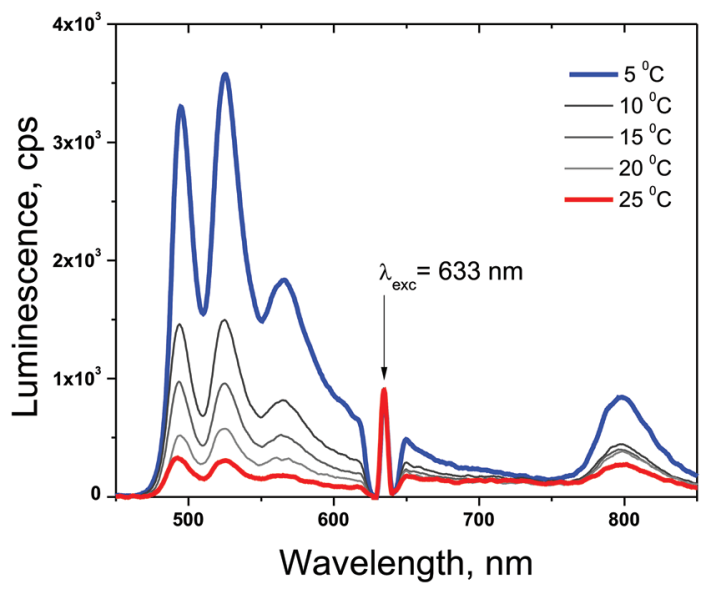

Fig. 4 Dependence of the delayed emitter fluorescence and residual sensitizer phosphorescence on the sample temperature. UC-couple PdTBP/BDP, dissolved in a mixture of $20 \%$ vol $\mathrm{N}$-butyl-2-pyridone/ toluene.
In Fig. 5 the temporal dependences of the UC-signal and residual sensitizer phosphorescence in an ambient environment for different sample temperatures are shown. It is clearly demonstrated that the ability of NBP to bind singlet oxygen decreases with a rise in the sample temperature. It is also evident that the delayed emitter fluorescence is much more sensitive to the local changes in the oxygen concentration than the residual phosphorescence: if the sample temperature is lower, this leads to an increase probability to bind and trap singlet oxygen, which cause a local decrease in the molecular oxygen concentration.

Despite the unique property to deliver temperature dependent protection of the excited triplet ensembles against singlet oxygen damage, NBP also demonstrates constraint potential to bind large amounts of singlet oxygen. Fig. 5 reveals the efficiency limitations of the NBP as a sacrificial singlet oxygen scavenger - even at a relatively large concentration $(20 \% \mathrm{vol})$, the total amount of the bound molecular oxygen can be estimated to be at least $200 \mu \mathrm{M}$ (saturation concentration of the oxygen in toluene ${ }^{31}$ ). Furthermore, in a bulk sample, after binding the present local amount of molecular oxygen (coinciding with the optically accessed spot) a strong increase
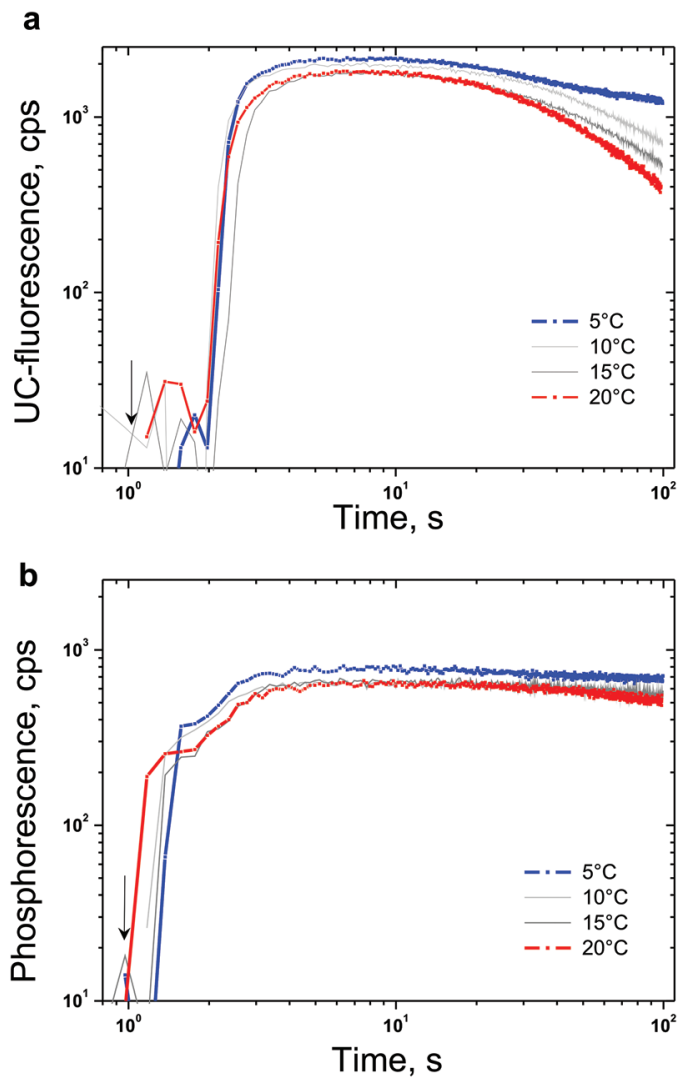

Fig. 5 (a) Temporal dependence of the UC-signal at the fluorescence maximum ( $\lambda=526 \mathrm{~nm}$ ) for different sample temperatures. (b) Temporal dependence of the residual sensitizer phosphorescence at the maximum $(\lambda=800 \mathrm{~nm})$ for different sample temperatures. Experimental conditions: the same as in Fig. 4. The start moment of optical excitation is marked by an arrow. 
in the UC-fluorescence is observed, since the optically accessed spot is a subject of increased molecular oxygen diffusion (from the non-excited regions) the newly arrived molecular oxygen will not be bound and a gradual loss of efficiency is observed first for UC-fluorescence, and afterwards for sensitizer residual phosphorescence (Fig. 5a and b, large observation times).

\section{Conclusions}

The temperature-dependent sacrificial singlet oxygen scavenging ability of $\mathrm{N}$-butyl-2-pyridone, ensuring efficient stimuliresponsive protection of densely populated excited triplet state ensembles against deactivation by molecular oxygen, was demonstrated. As an acting external stimulus the temperature was chosen: it was shown that at low temperature the concentration of singlet oxygen will be substantially lowered; in contrast, at elevated temperatures singlet oxygen will be not captured; thus the optically excited densely populated triplet ensembles will be effectively depopulated. The singlet oxygen scavenging ability of $\mathrm{N}$-butyl-2-pyridone demonstrates longterm protection of a triplet-triplet annihilation upconversion process against photooxidation. Additionally, the reported stimuli-responsive UC-process could be used as a real-time control for the concentration of singlet oxygen, generated during the post-illumination period for the purpose of fractional photodynamic therapy.

\section{Conflicts of interest}

The authors declare no competing financial interest or other potential conflict of interest.

\section{Acknowledgements}

This work is performed under European Horizon 2020 research and innovation programme under grant agreement no. 732794 - project HYPOSENS. S. Baluschev also acknowledges the DFNI E 02/11 - SunStore of the Bulgarian National Science Fund. Open Access funding provided by the Max Planck Society.

\section{Notes and references}

1 Y. Y. Cheng, B. Fueckel, T. Khoury, R. G. C. R. Clady, M. J. Y. Tayebjee, N. J. Ekins-Daukes, M. J. Crossley and T. W. Schmidt, J. Phys. Chem. Lett., 2010, 1, 1795-1799; S. K. Sugunan, C. Greenwald, M. F. Paige and R. P. Steer, J. Phys. Chem. A, 2013, 117, 5419-5427; X. Cao, B. Hu and P. Zhang, J. Phys. Chem. Lett., 2013, 4, 2334-2338; J. S. Lissau, D. Nauroozi, M. P. Santoni, S. Ott, J. M. Gardner and A. Morandeira, J. Phys. Chem. C, 2013, 117, 14493-14501.
2 P. R. Ogilby, Chem. Soc. Rev., 2010, 39, 3181-3209.

3 X. Wang, O. S. Wolfbeis and R. J. Meier, Chem. Soc. Rev., 2013, 42, 7834-7869.

4 A. Fercher, S. M. Borisov, A. V. Zhdanov, I. Klimant and D. B. Papkovsky, ACS Nano, 2011, 5, 5499-5508.

5 T. Spielmann, L. Xu, A. K. B. Gad, S. Johansson and J. Widengren, FEBS J., 2014, 281, 1317-1332.

6 C. Wu, B. Bull, K. Christensen and J. McNeill, Angew. Chem., Int. Ed., 2009, 48, 2741-2745.

7 T. V. Esipova, A. Karagodov, J. Miller, D. F. Wilson, T. M. Busch and S. A. Vinogradov, Anal. Chem., 2011, 83, 8756-8765.

8 J. A. Spencer, F. Ferraro, E. Roussakis, A. Klein, J. Wu, J. M. Runnels, W. Zaher, L. J. Mortensen, C. Alt, R. Turcotte, R. Yusuf, S. A. Vinogradov, D. T. Scadden and C. P. Lin, Nature, 2014, 508, 269-273.

9 K. Landfester, A. Svagan, J. Risbo and S. Balouchev, WO 2015/059179A1, EP 2865443A1, 2015; K. Landfester, M. Filatov, Yu. Avlasevich, D. Busko, A. Turshatov, F. Wurm, F. Marsico and S. Balouchev, EP 2851407A1, WO 2015/044129A1, 2015.

10 R. R. Islangulov, J. Lott, C. Weder and F. N. Castellano, J. Am. Chem. Soc., 2007, 129, 12652-12653; S. Baluschev, V. Yakutkin, T. Miteva, Y. Avlasevich, S. Chernov, S. Aleshchenkov, G. Nelles, A. Cheprakov, A. Yasuda, K. Mullen and G. Wegner, Angew. Chem., Int. Ed., 2007, 46, 7693-7696; S. Hoseinkhani, R. Tubino, F. Meinardi and A. Monguzzi, Phys. Chem. Chem. Phys., 2015, 17, 40204024.

11 J. Zhou, Q. Liu, W. Feng, Y. Sun and F. Li, Chem. Rev., 2015, 115, 395-465.

12 S. H. C. Askes, N. López Mora, R. Harkes, R. I. Koning, B. Koster, T. Schmidt, A. Kros and S. Bonnet, Chem. Commun., 2015, 51, 9137-9140; Ch. Wohnhaas, V. Mailänder, M. Dröge, M. A. Filatov, D. Busko, Y. Avlasevich, S. Baluschev, T. Miteva, K. Landfester and A. Turshatov, Macromol. Biosci., 2013, 13, 1422-1430; J. H. Kim and J. H. Kim, J. Am. Chem. Soc., 2012, 134, 17478-17481; Q. Liu, T. S. Yang, W. Feng and F. Y. Li, J. Am. Chem. Soc., 2012, 134, 5390-5397; C. Wohnhaas, A. Turshatov, V. Mailander, S. Lorenz, S. Baluschev, T. Miteva and K. Landfester, Macromol. Biosci., 2011, 11, 772-778; S. M. Borisov, R. Saf, R. Fischer and I. Klimant, Inorg. Chem., 2013, 52, 1206-1216; G. Massaro, J. Hernando, D. Ruiz-Molina, C. Roscini and L. Latterini, Chem. Mater., 2016, 28, 738-745.

13 K. Börjesson, P. Rudquist, V. Gray and K. Moth-Poulsen, Nat. Commun., 2016, 7, 12689; Z. Huang, X. Li, M. Mahboub, K. M. Hanson, V. M. Nichols, H. Le, M. L. Tang and C. J. Bardeen, Nano Lett., 2015, 15, 55525557; C. Mongin, S. Garakyaraghi, N. Razgoniaeva, M. Zamkov and F. N. Castellano, Science, 2016, 351, 369372.

14 T. F. Schulze, J. Czolk, Y. Y. Cheng, B. Fuckel, R. W. MacQueen, T. Khoury, M. J. Crossley, B. Stannowski, K. Lips and U. Lemmer, J. Phys. Chem. C, 2012, 116, 22794- 
22801; W. Q. Zou, C. Visser, J. A. Maduro, M. S. Pshenichnikov and J. C. Hummelen, Nat. Photonics, 2012, 6, 560-564; P. Duan, N. Yanai, H. Nagatomi and N. Kimizuka, J. Am. Chem. Soc., 2015, 137, 1887-1894; T. F. Schulze and T. W. Schmidt, Energy Environ. Sci., 2015, 8, 103-125; R. E. Keivanidis, S. Baluschev, G. Lieser and G. Wegner, ChemPhysChem, 2009, 10, 2316-2326.

15 M. I. J. Lorenz, S. H. Lorenz and O. S. Wolfbeis, Chem. Soc. Rev., 2010, 39, 3102.

16 M. A. Filatov, S. Baluschev and K. Landfester, Chem. Soc. Rev., 2016, 45, 4631-4852.

17 X. Cui, J. Zhao, Y. Zhou, J. Ma and Y. Zhao, J. Am. Chem. Soc., 2014, 136(26), 9256-9259; R. Tao, J. Zhao, F. Zhong, C. Zhang, W. Yang and K. Xu, Chem. Commun., 2015, 51, 12403-12406.

18 J. M. Aubry, C. Pierlot, J. Rigaudy and R. Schmidt, Acc. Chem. Res., 2003, 36, 668-675.

19 M. A. Filatov and M. O. Senge, Mol. Syst. Des. Eng., 2016, 1, 219-346.

20 H. H. Wasserman and J. R. Scheffer, J. Am. Chem. Soc., 1967, 89, 3073-3075.

21 M. A. Filatov, E. Heinrich, D. Busko, I. Z. Ilieva, K. Landfester and S. Baluschev, Phys. Chem. Chem. Phys., 2015, 17, 6501-6510.

22 I. S. Turan, D. Yildiz, A. Turksoy, G. Gunaydin and E. U. Akkaya, Angew. Chem., Int. Ed., 2016, 55, 28752878; S. Kolemen, T. Ozdemir, D. Lee, G. M. Kim,
T. Karatas, J. Yoon and E. U. Akkaya, Angew. Chem., Int. Ed., 2016, 55, 3606-3610; A. M. Asadirad, Z. Arno and N. R. Branda, Chem. Commun., 2013, 49, 56395641.

23 S. Mata, V. Cortijo, W. Caminati, J. L. Alonso, M. Sanz, J. C. Lopez and S. Blanco, J. Phys. Chem. A, 2010, 114, 11393-11398.

24 H. Tomisawa, H. Hongo, T. Hatano, H. Nakano and R. Fujita, Chem. Pharm. Bull., 1987, 35, 530-534.

25 S. Benz, S. Nötzli, J. S. Siegel, D. Eberli and H. J. Jessen, J. Med. Chem., 2013, 56, 10171-10182.

26 C. Changtong, D. W. Carney, L. Luo, C. A. Zoto, J. L. Lombardi and R. E. Connors, J. Photochem. Photobiol., A, 2013, 260, 9-13.

27 D. Saini, N. K. Rangra and P. C. Behra, Exploring the Biological Potential of Pyridones, Lambert Academic Publishing, 2015, ISBN-13:978-3-659-39505-5.

28 A. J. Svagan, D. Busko, Yu. Avlasevich, G. Glasser, S. Baluschev and K. Landfester, ACS Nano, 2014, 8, 81988820 .

29 S. McN. Sieburth, C. H. Lin and D. Rucando, J. Org. Chem., 1999, 64, 950-953.

30 K. Katta, D. Busko, Yu. Avlasevich, R. Muñoz-Espí, S. Baluschev and K. Landfester, Macromol. Rapid Commun., 2015, 36, 1084-1088.

31 Z. A. Li, S. Tang, P. Tan, C. Liu and B. Liang, J. Chem. Eng. Data, 2007, 52, 2339-2344. 